\title{
Harmonic Maps Between Noncompact Manifolds
}

\author{
Anestis Fotiadis
}

University of Warwick, $U K$

E-mail: riemann29@hotmail.com

\begin{abstract}
We describe the problem of finding a harmonic map between noncompact manifold. Given some sufficient conditions on the domain, the target and the initial map, we prove the existence of a harmonic map that deforms the given map.
\end{abstract}

\section{Introduction}

For a long time researchers have been trying to prove the existence of harmonic maps. In this paper the problem of how to deform an initial map into a harmonic map will be discussed in the case when both the domain and the target are noncompact. The first result in this direction has been obtained by Li and Tam in [14]. In the aforementioned paper the method applied is the heat flow method first introduced by Eells and Sampson in [8]. In the proof of the main theorems in this paper we make use of the compact exhaustion method. In particular, the proofs are simpler and the results are more general than the ones in [14].

\section{Background}

Let $M$ and $N$ be two Riemannian manifolds of dimension $m$ and $n$ respectively. Their metrics in local coordinates are written as

$$
d s_{M}^{2}=\sum_{k, j=1}^{m} g_{k j} d x^{k} d x^{j} \text { and } d s_{N}^{2}=\sum_{\alpha, \beta=1}^{n} h_{\alpha \beta} d x^{\alpha} d x^{\beta}
$$

respectively. Let $\left(g^{k j}\right)=\left(g_{k j}\right)^{-1}$ be the inverse metric tensor and $\Gamma_{k j}^{l}$ the Christoffel symbols for $M$, where the Latin indices $k, j, l$ take values from 1 to $m$. The determinant of the matrix $\left(g_{k j}\right)$ shall be denoted by $g$. We use the corresponding notation for the manifold $N$ but using Greek indices (from 1 to $n$ ) in this case.

Consider a $C^{1}$ map $u: M \rightarrow N$. The energy density of the map $u$ is defined in local coordinates by

$$
e(u)(x)=\frac{1}{2} \sum_{\alpha, \beta, k, j} g^{k j}(x) \frac{\partial u^{\alpha}}{\partial x^{k}} \frac{\partial u^{\beta}}{\partial x^{j}} h_{\alpha \beta}(u(x)) .
$$


Define

$$
E(u)=\int_{M} e(u) d v_{M}
$$

to be the energy of a $C^{1}$ map $u: M \rightarrow N$, where $d v_{M}$ is the volume form of $M$ and in local coordinates is given by $d v_{M}=\sqrt{g} d x^{1} \wedge \ldots \wedge d x^{m}$.

Since $E(u)$ is a real number (or infinity) for every $u$ in $C^{\infty}(M, N)$, it follows that the energy $E$ can be regarded as a functional.

If $u: M \rightarrow N$ is a smooth map, the tension field is a section of the pulled back bundle $u^{-1} T N$ which is given intrinsically by

$$
\tau(u)=\operatorname{Tr}(\nabla d u) .
$$

In local coordinates,

$$
\begin{aligned}
\tau^{\alpha}(u)(x) & =g^{k j}(x) \frac{\partial^{2} u^{\alpha}}{\partial x^{k} \partial x^{j}}(x)-g^{k j}(x) \Gamma_{k j}^{l}(x) \frac{\partial u^{\alpha}}{\partial x^{l}}(x) \\
& +g^{k j}(x) \Gamma_{\beta \gamma}^{\alpha}(u(x)) \frac{\partial u^{\beta}}{\partial x^{k}}(x) \frac{\partial u^{\gamma}}{\partial x^{j}}(x) \\
& =\Delta_{M} u^{\alpha}(x)+g^{k j}(x) \Gamma_{\beta \gamma}^{\alpha}(u(x)) \frac{\partial u^{\beta}}{\partial x^{k}}(x) \frac{\partial u^{\gamma}}{\partial x^{j}}(x),
\end{aligned}
$$

where $\Delta_{M}$ is the Laplace Beltrami operator of $(M, g)$.

Definition 2.1. A map is called harmonic if its tension field vanishes identically.

The harmonic maps are the critical points of the energy functional with respect to compactly supported variations (see [13] for more details).

In what follows, we discuss the problem of how to deform a given map into a harmonic map when both the domain and the target are noncompact. The issue of extending a given boundary map to a map with the required properties, will be discussed in a forthcoming paper.

In the next section there are results that involve some integral estimates on the norm of the tension field of the given map. The norm symbol $\|\tau(\Phi)\|(x)$ denotes the pointwise norm given by the inner product $h(\Phi(x))(\tau(\Phi)(x), \tau(\Phi)(x))$.

We apply the method of compact exhaustion, as in the work of Schoen and Yau in [16]. Let $B_{R}(x)$ be the geodesic ball in $M$ with center $x$ and radius $R>0$. The following local gradient estimate for harmonic maps was proved by Cheng in [4], and it is applied in the proofs of this paper.

Proposition 2.2 (Cheng). Consider $N$ to be a simply connected Riemannian manifold with nonpositive sectional curvature, and let $M$ be a complete Riemannian manifold. Let $u: M \rightarrow N$ be $a$ harmonic map and assume that it maps the geodesic ball $B_{2}\left(x_{0}\right)$, into a geodesic ball $B_{R}\left(y_{0}\right)$. Then,

$$
\sup _{x \in B_{1}\left(x_{0}\right)} e(u)(x) \leq C,
$$

for a constant $C$ depending on $m, R$ and $K \geq 0$, where $\operatorname{Ric}(M) \geq-K$ on $B_{2}\left(x_{0}\right)$. 


\section{The Heat Flow Method}

Partial differential equations for maps between manifolds are of considerable interest. An important example is the harmonic map equation introduced for manifolds by Eells and Sampson in [8]. In this section we give a presentation of the heat flow method.

Given a map $\Phi$ consider a one-parameter family $u_{t}: M \rightarrow N$ deforming $\Phi$. The aim is to construct $u_{t}$, in such a way that it converges to a harmonic map $u_{\infty}$ as $t \rightarrow+\infty$.

Definition 3.1. The map $u: M \times[0, T) \rightarrow N$ solving the parabolic initial boundary value problem

$$
\begin{aligned}
\frac{\partial u}{\partial t}(x, t) & =\tau(u)(x, t) \text { on } M \times[0, T) \\
u(x, 0) & =\Phi(x) \text { on } M \times\{0\} \\
u(x, t) & =\Phi(x) \text { on } \partial M \times[0, T)
\end{aligned}
$$

for some positive (possibly infinite) $T$, is called the heat flow and $\Phi$ the initial map. The variables $x$ and $t$ are usually referred to as the space and time variables respectively.

Note that when $\partial M=\emptyset$ the last equation above holds trivially. Eells and Sampson, and later Hamilton, established that given a $C^{2+\alpha}$ map $\Phi: M \rightarrow N$, then there exists a $T>0$ depending on $\Phi$, and the geometry of the domain and target, such that the heat flow exists on $M \times[0, T)$ (see for example [12] or [8]).

The kinetic energy density of a map $u: M \times[0,+\infty) \rightarrow N$ is defined by

$$
\kappa(u)=\frac{1}{2}\left\|\frac{\partial u}{\partial t}\right\|^{2}=\frac{1}{2}\left\langle\frac{\partial u}{\partial t}, \frac{\partial u}{\partial t}\right\rangle .
$$

In local coordinates,

$$
\kappa(u)(x, t)=\frac{1}{2} \frac{\partial u^{\alpha}}{\partial t} \frac{\partial u^{\beta}}{\partial t} h_{\alpha \beta}(u(x, t)) .
$$

Define

$$
K(u)=\int_{M} \kappa(u) d v_{M}
$$

as the kinetic energy of $u$. As in the case of the energy, the kinetic energy can be regarded as a functional.

From now on $u$ will denote the heat flow and $\Phi$ the initial map. On the assumption that a heat flow exists for all positive time, the kinetic energy density satisfies the following equation

$$
\frac{\partial \kappa}{\partial t}=\Delta \kappa-\left\|\nabla \frac{\partial u}{\partial t}\right\|^{2}+\left\langle R_{N}(u)\left(d u\left(e_{j}\right), \frac{\partial u}{\partial t}\right) d u\left(e_{j}\right), \frac{\partial u}{\partial t}\right\rangle .
$$

The energy density of $u$ satisfies the following equation

$$
\begin{aligned}
\frac{\partial e(u)}{\partial t} & =\Delta e(u)-\|\nabla d u\|^{2}-\left\langle d u\left(\operatorname{Ric}_{M}\left(e_{k}, e_{j}\right) e_{j}\right), d u\left(e_{k}\right)\right\rangle \\
& +\left\langle R_{N}\left(d u\left(e_{k}\right), d u\left(e_{j}\right)\right) d u\left(e_{j}\right), d u\left(e_{k}\right)\right\rangle,
\end{aligned}
$$


where $\operatorname{Ric}_{M}, R_{N}$ and $\Delta$ are the Ricci tensor of $M$, the curvature tensor of $N$ and the Laplacian operator of $M$ respectively. The vectors $e_{k}, k=1,2, \ldots, m$ represent an orthonormal frame on $M$, as usual. These are known as the Weitzenböck formulas for $e(u)$ and $\kappa(u)$ respectively, a proof of which can be found in [15].

Observe that all the above formulas hold, with the time derivative terms omitted, when $u$ is a harmonic map. This is a consequence of the fact that a harmonic map is a time independent heat flow.

Let $\widehat{M}$ and $\widehat{N}$ be the universal covers of $M$ and $N$ respectively, and let $\pi_{M}$ and $\pi_{N}$ be the relevant projection maps. Consider $\widehat{M}$ and $\widehat{N}$ equipped with the metrics from $M$ and $N$ pulled back by the projection maps. Then $M=\widehat{M} / F_{M}$ and $N=\widehat{N} / F_{N}$, where $F_{M}$ and $F_{N}$ the group of deck transformations of $M$ and $N$ respectively. Let $U: M \times[0, T] \rightarrow N$ be a homotopy of $\Phi$ and $u$. In particular, take $U$ to be the heat flow. Choose a lifting $\widehat{U}: \widehat{M} \times[0, T] \rightarrow \widehat{N}$. Then, there exists a homomorphism $h: F_{M} \rightarrow F_{N}$ independent of $t$ such that $\widehat{U}(g(y), t)=h(g)(\widehat{U}(y, t))$, for every $t \in[0, T], g \in F_{M}$ and $y \in \widehat{M}$. Let $\widehat{\Phi}(y)=\widehat{U}(y, 0)$ and $\widehat{u}(y)=\widehat{U}(y, T)$. Denote by $d_{\widehat{N}}$ the distance function on $\widehat{N}$ and observe, that from the above it follows that $d_{\widehat{N}}(\widehat{\Phi}(y), \widehat{u}(y))$ is $F_{M}$ invariant.

Define the function $\rho(\Phi, u)$ on $M$ by $\rho(\Phi, u)(x)=d_{\widehat{N}}(\widehat{\Phi}(y), \widehat{u}(y))$, where $\pi_{M}(y)=x$. Then, according to the above, $\rho$ is a well defined function. Note that $\rho(\Phi, u)(x) \geq d_{N}(\Phi(x), u(x))$. If $N$ is simply connected, then the lifting of the heat flow is no more necessary and in such a case $\rho(\Phi, u)(x)=d_{N}(\Phi(x), u(x))$ holds.

From now on we assume that $N$ has non-positive sectional curvature. This implies that $d_{\widehat{N}}$ is smooth on $\widehat{N} \times \widehat{N}$ except on the diagonal. Let $x$ in $M$ and $e_{j}$ be an orthonormal frame near $x$, where $j=1,2, \ldots, m$. Fix $t$ and take orthonormal frames $f_{\alpha}$ and $\bar{f}_{\alpha}$ near $\widehat{u}(y, t)$ and $\widehat{\Phi}(y)$ respectively, where $\alpha=1,2, \ldots, n$. If $d \widehat{u}\left(e_{j}\right)=\sum_{\alpha} \widehat{u}_{j}^{\alpha} f_{\alpha}$ and $d \widehat{\Phi}\left(e_{j}\right)=\sum_{\alpha} \widehat{\Phi}_{j}^{\alpha} \bar{f}_{\alpha}$, then $X_{j}=\widehat{u}_{j}^{\alpha} f_{\alpha}+\widehat{\Phi}_{j}^{\alpha} \bar{f}_{\alpha}$ is a vector in the tangent space of $\widehat{N} \times \widehat{N}$ at $(\widehat{u}, \widehat{\Phi})$. If $r: \widehat{N} \times \widehat{N} \rightarrow[0,+\infty)$ is the distance function, then the Hessian of $r$ is $r_{X_{k} X_{j}}=X_{j} X_{k}(r)-\left(\nabla_{X_{j}} X_{k}\right)(r)$. It follows, as in [5] that

$$
\begin{aligned}
\frac{\partial \rho}{\partial t} & =\Delta \rho-\sum_{j} r_{X_{j} X_{j}}-\sum_{j, \alpha} r_{\alpha} \widehat{\Phi}_{j j}^{\alpha} \\
& \leq \Delta \rho-\sum_{j} r_{X_{j} X_{j}}+\|\tau(\Phi)\| .
\end{aligned}
$$

Taking into account that $N$ has non-positive sectional curvature it follows by the work of Schoen and Yau in [17] that Hessian terms $r_{X_{j} X_{j}}$ are non-negative. Thus,

$$
\frac{\partial \rho}{\partial t} \leq \Delta \rho+\|\tau(\Phi)\|
$$

holds on $M$, except at the points where $\rho(\Phi(x), u(x, t))=0$. In fact, formula (3.6) holds on $M$ in the distributional sense, as shown in [5] by Ding and Wang.

\section{Extending the Result of Donnelly}

The main result in the theory of harmonic maps is the existence theorem of Eells and Sampson [8], with the extensions of Hamilton [12], and is as follows. 
Theorem 4.1 (Eells, Sampson and Hamilton). Consider $M$ and $N$ to be compact Riemannian manifolds with (possibly empty) boundaries. In addition take $N$ to have non-positive sectional curvature. Then, every smooth map from $M$ to $N$ is homotopic to a harmonic map.

One would like to find harmonic maps without having to assume that the domain and target are compact. Avilés, Choi and Micallef in [2] succeeded in doing this, by considering maps of bounded image. They regard the harmonic map problem, as a perturbation of the corresponding problem of real valued continuous functions, which has been solved by Anderson and Schoen in [1]. A variant of their proof, which is similar to the proofs in the present paper, has been given by Donnelly in [6]. He considers the case of $C^{2}$ maps $\Phi: M \rightarrow N$ of bounded image, where $M$ is a complete Riemannian manifold, which admits a Green's function $G(x, y)$ and $N$ is a complete simply connected manifold with non-positive curvature. A similar result has been proved by Bando in [3] .

Theorem 4.2 (Donnelly). Consider $N$ to be a complete simply connected Riemannian manifold with non-positive sectional curvature and $M$ to be a complete Riemannian manifold which admits a Green's function. Then, every $C^{2}$ map $\Phi: M \rightarrow N$ is at a bounded distance from a harmonic map $u: M \rightarrow N$, provided that the following integral

$$
w(x)=\int_{M} G(x, y)\|\tau(\Phi)\|(y) d v_{M},
$$

is uniformly bounded.

Proof. Take $M_{j}, j \in \mathbb{N}$, to be a compact exhaustion of $M$ by smooth domains. Define $\Phi_{j}$ as the map $\Phi$ restricted to $M_{j}$. Fix $j$ and take into account the result of Hamilton [12] and that $N$ has negative sectional curvature. It follows that there is a harmonic map $u_{j}: M_{j} \rightarrow N$ that is continuous up to the boundary such that $u_{j}=\Phi_{j}$ on $\partial M_{j}$. Let $d_{j}=d\left(u_{j}, \Phi_{j}\right)$, where $d$ denotes the distance function.

By the work of Schoen and Yau in [17], it follows that

$$
\Delta d_{j} \geq-\left\|\tau\left(\Phi_{j}\right)\right\| \geq-\|\tau(\Phi)\|=\Delta w,
$$

where $w$ is the function given by $w(x)=\int_{M} G(x, y)\|\tau(\Phi)\|(y) d y$ which by assumption is uniformly bounded. Then, the function $v_{j}(x)=d_{j}(x)-w(x), x \in M_{j}$, satisfies $\Delta v_{j}(x) \geq 0$ for every $x \in M_{j}$ and $v_{j}\left(x_{0}\right) \leq 0$ for every $x_{0} \in \partial M_{j}$. Hence, applying the maximum principle, it follows that $v_{j} \leq 0$ and thus $d_{j} \leq w$ everywhere in $M_{j}$. This result, together with the assumption on $w$ imply that $d_{j} \leq C$, where $C$ is a positive constant independent of $j$. By the triangular inequality, it follows that

$$
\begin{aligned}
d_{j}\left(u_{j}(x), u_{j}(y)\right) & \leq d_{j}\left(\Phi(x), u_{j}(x)\right)+d_{j}(\Phi(x), \Phi(y))+d_{j}\left(\Phi(y), u_{j}(y)\right) \\
& \leq 2 C+\sqrt{2 \sup _{w \in M_{j}} e(\Phi)(w)} d(x, y) .
\end{aligned}
$$

Using the estimates of Cheng in [4] we find that the energy density $e\left(u_{j}\right)(x)$ is bounded (with a bound depending on $j$ ) for all $x$ such that $B_{2}(x) \subset M_{j}$. Note that by definition, $u_{j}$ maps $M_{j}$ to $\Phi\left(M_{j}\right)$ and that $d_{j}\left(u_{j}(x), u_{j}(y)\right) \leq \sqrt{2 \sup _{w \in M_{j}} e\left(u_{j}\right)(w)} d(x, y)$. By the uniform bounds on the gradient of $u_{j}$ on each compact set $K \subset M$ and taking into account the standard results for linear elliptic equations in [9] it follows that there are uniform bounds for the higher derivatives of $u_{j}$ 
on compact sets $K$. Applying the Arzela-Ascoli theorem, we find a subsequence $j_{k}$, such that $u_{j_{k}}$ converges uniformly on compact sets to a harmonic map $u$ that is a bounded distance from $\Phi$ (see p.4 in [6]). This completes the proof.

Donnelly in [6] proved that the integral $w(x)=\int_{\mathbb{H}^{m}} G(x, y)\|\tau(\Phi)\|(y) d y$ is uniformly bounded for every $C^{2}$ map $\Phi: \mathbb{H}^{m} \rightarrow \mathbb{H}^{n}$ with bounded image, and thus he recovers the next result of Avilés, Choi and Micallef.

Theorem 4.3 (Avilés, Choi and Micallef). Every $C^{2}$ map $\Phi: \mathbb{H}^{m} \rightarrow \mathbb{H}^{n}$, that is continuous up to the ideal boundary and of bounded image, is at a bounded distance from a harmonic map $u: \mathbb{H}^{m} \rightarrow \mathbb{H}^{n}$,

Recall that if $N$ is not simply connected, then the equation $0 \leq \Delta \rho+\|\tau(\Phi)\|$ holds in the distributional sense (see [5] for a proof) and $\rho$ is smooth everywhere except on the diagonal. We use this last formula and the maximum principle (that is the first and second derivative tests from calculus, see [12] for more details) to extend the result of Theorem 4.2. The maximum principle can only be used everywhere except the diagonal where $\rho$ is not smooth, but in the diagonal $\rho$ is 0 (thus bounded). The next more general theorem holds.

Theorem 4.4 (Extended Result of Donnelly). Let $N$ be a complete Riemannian manifold with nonpositive sectional curvature. Let $M$ be a complete Riemannian manifold which admits a Green's function. Then, every $C^{2}$ map $\Phi: M \rightarrow N$ is at a bounded distance from a harmonic map $u: M \rightarrow N$ provided that the following integral

$$
w(x)=\int_{M} G(x, y)\|\tau(\Phi)\|(y) d v_{M}
$$

is uniformly bounded.

Proof. Take $M_{j}, j \in \mathbb{N}$, to be a compact exhaustion of $M$. Define $\Phi_{j}$ as the map $\Phi$ restricted to $M_{j}$. Fix $j$ and solve the relevant Dirichlet problem in $M_{j}$ and let $u_{j}$ be the harmonic map homotopic to $\Phi_{j}$. Use equation $0 \leq \Delta \rho+\|\tau(\Phi)\|$ and the same approach as in the proof of Theorem 4.2, in order to prove that $\rho_{j}$ is uniformly bounded, where $\rho_{j}=\rho\left(u_{j}, \Phi_{j}\right)$ is the distance function as in [17]. Since $d\left(u_{j}, \Phi_{j}\right) \leq \rho_{j}\left(u_{j}, \Phi_{j}\right)$, it follows that the distance of $u_{j}$ from $\Phi_{j}$ is also uniformly bounded. Observe that $e(u)(x)=e(\widehat{u})(y)$, where $\widehat{u}: \widehat{M} \rightarrow \widehat{N}$ is a lift of $u$. The estimate of Cheng then implies that the energy density $e\left(u_{j}\right)(x)$ is bounded (with a bound depending on $j$ ) for all $x$ such that $B_{2}(x) \subset M_{j}$. Taking into account the standard results for linear elliptic equations in [9] it follows that there are uniform bounds for the higher derivatives of $u_{j}$ on each compact set $K \subset M$. Applying the Arzela-Ascoli theorem, we find a subsequence $j_{k}$, such that $u_{j_{k}}$ converges uniformly on compact sets to a harmonic map $u$ that is at a bounded distance from $\Phi$ (see p.4 in [6] for a similar argument). This completes the proof of the theorem.

\section{Extending Results of Li and Tam}

In [14] Li and Tam published some general results on the harmonic map problem when both the domain and the target manifolds are noncompact. Their work is motivated by the work of Eells and Sampson in [8] and of Hamilton in [12]. They use the heat flow equation, in order to deform a given initial map (defined between noncompact manifolds in this case) into a harmonic map. 
A proof using a compact exhaustion is applied to provide similar, but more general, results to these in [14]. Instead of the heat flow, we only need the properties of the heat kernel.

We say that a manifold has bounded geometry, if its Ricci curvature is bounded below and its injectivity radius is positive.

Theorem 5.1 (Li and Tam). Let $N$ be a complete simply connected Riemannian manifold with nonpositive sectional curvature and $M$ be a complete Riemannian manifold with bounded geometry and positive lower bound of the spectrum. Then, for every $C^{2}$ map $\Phi: M \rightarrow N$, a harmonic map $u: M \rightarrow N$ exists that is at a bounded distance from $\Phi$, provided that $\|\tau(\Phi)\|^{2}$ is in $L^{p}(M)$ for some $p \in(1, \infty)$.

New, short proof. Firstly recall that from Theorem 4.2, it is enough to show that

$$
w(x)=\int_{M} G(x, y)\|\tau(\Phi)\|(y) d v
$$

is uniformly bounded, where $G$ is the Green's function of $M$.

Using that $G(x, y)=\int_{0}^{+\infty} H(x, y, t) d t$, and changing the order of integration, it follows that it is enough to show that

$$
w(x)=\int_{0}^{+\infty} \int_{M} H(x, y, t)\|\tau(\Phi)\|(y) d v
$$

is uniformly bounded. Hence, it suffices to show that the integral

$$
\int_{M} H(x, y, t)\|\tau(\Phi)\|(y) d v
$$

decays exponentially to zero, as $t \rightarrow+\infty$. From the Markov property of the heat kernel $\int_{M} H(x, y, t) d v=$ 1 (see for example [7] for more details). Applying the Hölder inequality, it follows that

$$
\begin{aligned}
\int_{M} H(x, y, t)\|\tau(\Phi)\|(y) d v & =\int_{M} H^{\frac{1}{2}}(x, y, t) H^{\frac{1}{2}}(x, y, t)\|\tau(\Phi)\|(y) d v \\
& \leq\left\{\int_{M} H(x, y, t)\|\tau(\Phi)\|^{2}(y) d v\right\}^{\frac{1}{2}} .
\end{aligned}
$$

Thus, it is enough to show that

$$
\int_{M} H(x, y, t)\|\tau(\Phi)\|^{2}(y) d v
$$

decays exponentially to zero, as $t \rightarrow+\infty$. Applying the Hölder inequality again, it follows that

$$
\int_{M} H(x, y, t)\|\tau(\Phi)\|^{2}(y) d v \leq\left(\int_{M}\|\tau(\Phi)\|^{2 p}(y) d v\right)^{\frac{1}{p}} \cdot\left(\int_{M} H^{q}(x, y, t) d v\right)^{\frac{1}{q}} .
$$

Then the result follows from the hypothesis on $\|\tau(\Phi)\|$ and the next lemma, a proof of which can be found in [14].

For the proof of Theorem 5.1 we need the following lemma. 
Lemma 5.2. Let $q>1$ and $M$ a Riemannian manifold with bounded geometry and positive lower bound of the spectrum. Then, there exists a constant $C$ depending on $q, m, \inf _{x \in M} \operatorname{Vol}\left(B_{1}(x)\right)$ and the lower bound of the Ricci curvature, such that for every $x$ in $M$ and for every $t>1$ the following estimate holds,

$$
\int_{M} H^{q}(x, y, t) d v \leq C \exp \left(-\frac{4 \lambda_{0}(M)(q-1) t}{q}\right),
$$

where $\lambda_{0}(M)$ is the lower bound of the spectrum of the manifold $M$.

Remark: Note that the same proof as above, without having to consider the inequality

$$
\begin{aligned}
\int_{M} H(x, y, t)\|\tau(\Phi)\|(y) d v & =\int_{M} H^{\frac{1}{2}}(x, y, t) H^{\frac{1}{2}}(x, y, t)\|\tau(\Phi)\|(y) d v \\
& \left.\leq \int_{M} H(x, y, t)\|\tau(\Phi)\|^{2}(y) d v\right\}^{\frac{1}{2}} .
\end{aligned}
$$

shows that $\int_{M} H(x, y, t)\|\tau(\Phi)\|(y) d v$ decays exponentially to zero, as time progresses to infinity, provided that $\|\tau(\Phi)\|$ is in $L^{p}(M)$ for some $p \in(1,+\infty)$. An application to the case of hyperbolic spaces thus provides the following theorem that is not covered by the results of Li and Tam in [14].

Theorem 5.3. For every $C^{2}$ map $\Phi: \mathbb{H}^{m} \rightarrow \mathbb{H}^{n}$ there exists a harmonic map $u: \mathbb{H}^{m} \rightarrow \mathbb{H}^{n}$ that is at a bounded distance from $\Phi$, provided that $\|\tau(\Phi)\|$ is in $L^{p}\left(\mathbb{H}^{m}\right)$ for some $p \in(1,+\infty)$.

The same approach as in Theorem 4.4 provides a proof of the following more general result that covers the case when $N$ is not simply connected. This new result is as follows.

Theorem 5.4. Let $N$ be a complete Riemannian manifold with non-positive sectional curvature. Let $M$ be a complete Riemannian manifold with bounded geometry and positive lower bound of the spectrum. Then, for every $C^{2}$ map $\Phi: M \rightarrow N$, there exists a harmonic map $u: M \rightarrow N$, that is at a bounded distance from $\Phi$, provided that $\|\tau(\Phi)\|^{2}$ is in $L^{p}(M)$ for some $p \in(1,+\infty)$.

Remark: If in addition the energy density of $\Phi$ is uniformly bounded then the energy density of $u$ is uniformly bounded. This is easy to find applying Proposition 2.2 .

Acknowledgments. I would like to thank Vlad Markovic and Mario Micallef for their advice and encouragement. I would like to thank Michel Marias for having reviewed the revised version of my paper.

\section{References}

[1] Anderson M T and Schoen R, Positive Harmonic Functions on Complete Manifolds of Negative Curvature, Ann. Math. 121 (1985), 429-461.

[2] Avilés P, Choi H and MiCAllef M, Boundary Behavior of Harmonic Maps on Non-smooth Domains and Complete Negatively Curved Manifolds, J. Funct. Anal. 99 (1991), 293-331.

[3] Bando S, The Existence Theorem of Harmonic Objects Via Green Function, Preprint.

[4] Cheng S Y, Liouville Theorem for Harmonic Maps of Complete Noncompact Riemannian Manifolds, Proc. Symp. Pure Math. 36 (1980), 147-151. 
[5] Ding W-Y and Wang Y, Harmonic Maps of Complete Noncompact Riemannian Manifolds, Int. J. Math. 2 (1991), 617-633.

[6] Donnelly H, Asymptotic Dirichlet Problem for Harmonic Maps with Bounded Image, Geometricae Dedicata 91 (2002), 1-6.

[7] DodzIUK J, Maximum Principle for Parabolic Inequalities and the Heat Flow on Open Manifolds, Indiana Univ. Math. J. 33 (1983), 703-716.

[8] Eells J and SAMPSON J, Harmonic mappings of Riemannian manifolds, Amer. J. Math. 86 (1964), $109-160$.

[9] Gilbarg D and Trudinger N, Elliptic Partial Differential Equations of Second Order, Grundlehren Math. Wiss. 224 (1983).

[10] GRIGOR'YAN A, Estimates of Heat Kernels on Riemannian Manifolds, www.ma.ic.ac.uk/ grigor.

[11] GRIGOR'YAN A, Heat Kernel on a non-Compact Riemannian Manifold,www.ma.ic.ac.uk/ grigor.

[12] Hamilton R S, Harmonic Maps of Manifolds with Boundary, Springer-Verlag (1975).

[13] Jost J, Riemannian Geometry and Geometric Analysis, (Third Edition) Springer (2002).

[14] LI P and TAM L-F, The heat equation and harmonic maps of complete manifolds, Invent. Math. 105 (1991), 1-46.

[15] Nishikawa S, Variational Problems in Geometry, Amer. Math. Soc. (2002).

[16] Schoen R and YAU S T, Harmonic Maps and the Topology of Stable Hypersurfaces and Manifolds with Non-Negative Ricci Curvature, Comment. Math. Helvetici 39 (1976), 333-341.

[17] SCHOEN R and YAU S T, Compact Group Action and the Topology of Manifolds with Nonpositive Curvature, Topology 18 (1979), 361-380. 